\title{
The Osteoporotic Condition as a Predictive Factor for Hemorrhagic Transformation in Acute Cardioembolic Stroke
}

\author{
Yu Deok Won, Jae-Min Kim, Je-Il Ryu, ${ }^{1}$ Seong-Ho Koh, ${ }^{2}$ Myung-Hoon Han, ${ }^{1}$ Jin-Hwan Cheong ${ }^{1}$ \\ Department of Neurosurgery, Hanyang University Guri Hospital, Guri, Korea \\ Department of Neurology, ${ }^{2}$ Hanyang University Guri Hospital, Guri, Korea
}

Objective : Hemorrhagic transformation $(\mathrm{HT})$ can be occurred after acute cerebral infarction. $\mathrm{HT}$ can worse symptoms in severe cases and adversely affect long-term prognosis. As bone and vascular smooth muscle are composed of type 1 collagen, we aimed to identify a potential relationship between bone mineral density (BMD) and $\mathrm{HT}$ after acute cardioembolic stroke.

Methods : As an indicator of BMD, we used mean frontal skull Hounsfield unit (HU) values on brain computed tomography (CT). Multivariative hazard ratios were calculated using Cox regression analysis to identify whether the osteoporotic condition was an independent predictor of $\mathrm{HT}$ after acute cardioembolic stroke.

Results : This 11-year analysis enrolled 506 patients who diagnosed as acute cardioembolic infarction. The first tertile of skull HU value was an independent predictor of HT development compared to the third tertile (hazard ratio, 2.12; $95 \%$ confidence interval, $1.13-3.98 ; p=0.020$ ). We observed no interactions between age and skull $\mathrm{HU}$ with respect to $\mathrm{HT}$ statistically.

Conclusion : The results of this study revealed an association between osteoporotic conditions and HT development after acute cardioembolic stroke. A convenient method to measure the cancellous bone $\mathrm{HU}$ value of the frontal skull using brain $\mathrm{CT}$ images may be useful for predicting $\mathrm{HT}$ in patients with acute cerebral infarction.

Key Words : Bone density · Hemorrhagic transformation · Skull · Embolic stroke.

\section{INTRODUCTION}

Hemorrhagic transformation (HT) can be occurred after acute cerebral infarction. Autopsy studies have shown that HT occurs in $18-42 \%$ of cases $^{19,22)}$. HT can worse symptoms in se- vere cases and adversely affect long-term prognosis. Therefore, the study of predictive factors of HT is clinically important.

Both bone and vessel smooth muscles are composed of type 1 collagen. Osteoporosis is a systemic disease that is strongly associated with the genetic components of type 1 collagen $^{11}$.

- Received : June 15, $2021 ・$ Revised : June 30, $2021 ・$ Accepted : July 5, 2021

- Address for reprints : Jin-Hwan Cheong

Department of Neurosurgery, Hanyang University Guri Hospital, 153 Gyeongchun-ro, Guri 11923, Korea

Tel : +82-31-560-2324, Fax : +82-31-560-2327, E-mail : cjh2324@hanyang.ac.kr, ORCID : https://orcid.org/0000-0002-0022-312X

\section{Myung-Hoon Han}

Department of Neurosurgery, Hanyang University Guri Hospital, 153 Gyeongchun-ro, Guri 11923, Korea

Tel : +82-31-560-2328, Fax : +82-31-560-2327, E-mail : gksmh80@gmail.com, ORCID : https://orcid.org/0000-0003-1728-5017

This is an Open Access article distributed under the terms of the Creative Commons Attribution Non-Commercial License (http://creativecommons.org/licenses/by-nc/4.0) which permits unrestricted non-commercial use, distribution, and reproduction in any medium, provided the original work is properly cited. 
Cancellous bone structures in the skull may be affected by osteoporotic conditions; thus, we assumed that low bone mineral density (BMD) would negatively affect vessel stability. Moreover, this mechanism may be associated with HT after acute cerebral infarction. We hypothesized that, this mechanism may be associated with HT development after acute cerebral infarction. Patients treated for acute cerebral infarction usually do not an examination of BMD during hospitalization, so we needed another way to predict osteoporosis. As we previously showed an association between the mean frontal skull Hounsfield unit (HU) value and T score ${ }^{13,26)}$, we used the frontal skull HU values instead of the T-score in the present study.

The overall objective of the study was to identify a possible relationship between BMD and HT after acute cerebral infarction. HT frequency and severity are reportedly high in patients with acute cerebral infarction due to cardiac embolism ${ }^{23)}$. Therefore, we included only cardioembolic stroke patients in this study to reduce the effects of heterogeneity among the various subtypes of ischemic stroke on our results.

\section{METERIALS AND METHODS}

This study was approved by the Institutional Review Board of Hanyang University Guri Hospital (IRB No. 2020-08-032) and conformed to the tenets of the Declaration of Helsinki, which waived the need for informed consent because of the retrospective nature of the study. All individual records were anonymized before the analysis.

\section{Patient selection}

We retrospectively collected data on all consecutive patients with acute cerebral infarction due to cardiac embolism from the Registry of Stroke Patients of our institute from January 1, 2009, to December 31, 2019. All stroke subtypes were classified according to the Trial of ORG 10172 in Acute Stroke Treatment (TOAST) classification ${ }^{1)}$. As we described in the INTRODUCTION, patients with acute cerebral infarction caused by other causes except cardiac embolism, were excluded to reduce the possible effects of heterogeneity on HT after acute cerebral infarction.

Overall, 578 patients were initially selected. Of these, 72 were excluded for 1) absence of follow-up computed tomogra- phy (CT) (because we need to measure the $\mathrm{HU}$ value using the CT image; 54 patients) and 2) no measurable cancellous bone (too narrow of an intercortical space of the frontal skull; 18 patients). The remaining 506 patients were included in this study. All patients were confirmed to have had a cardiac embolism infarction on brain magnetic resonance imaging (MRI), including diffusion-weighted imaging (DWI), electrocardiogram, laboratory studies, carotid Doppler imaging, and echocardiography.

\section{Baseline and follow-up images}

Brain CT images were obtained using CT scanner (Siemens SOMATOM Definition Edge or Siemens SOMATOM Definition AS; Seimens Healthcare, Forchheim, Germany) and DWI with an MRI scanner (Philips Ingenia 3.0T or Philips Achieva 3.0T; Philips Healthcare, Best, The Netherlands). All CT images were obtained with continuous slices, no gap, and 4.05.0-mm slice thickness. The duration (days) between the occurrence of acute cerebral infarction and the last follow-up CT images were investigated in all patients. In the patients had $\mathrm{HT}$, we measured the days from acute cardioembolic stroke onset to the CT at which HT was firstly observed.

\section{Measurement of the infarction volume and the skull HU values}

The infarction volume was measured using $\mathrm{ABCD} / 2$, a method of measuring intracerebral hemorrhage volume ${ }^{16,28)}$ using DWI images from MRI performed at admission. The DWI image with the largest area of acute infarction was selected. The maximum diameter (A in Fig. 1) of the infarction was measured. The largest diameter perpendicular to A was measured in the same image ( $B$ in Fig. 1). Then, the number of images with infarction was counted ( $\mathrm{C}$ in Fig. 1), and the thickness of the slices was measured (D in Fig. 1) (Fig. 1). We measured the frontal skull HU values in the cancellous bone using the "Linear histogram graph" function as previous described $^{13,31)}$. All CT images were magnified for HU measurement to minimize measurement errors, to avoid including cortical bone (Fig. 2). All frontal skull HUs were measured by two faculty neurosurgeons who were blinded to the clinical data of all patients. 


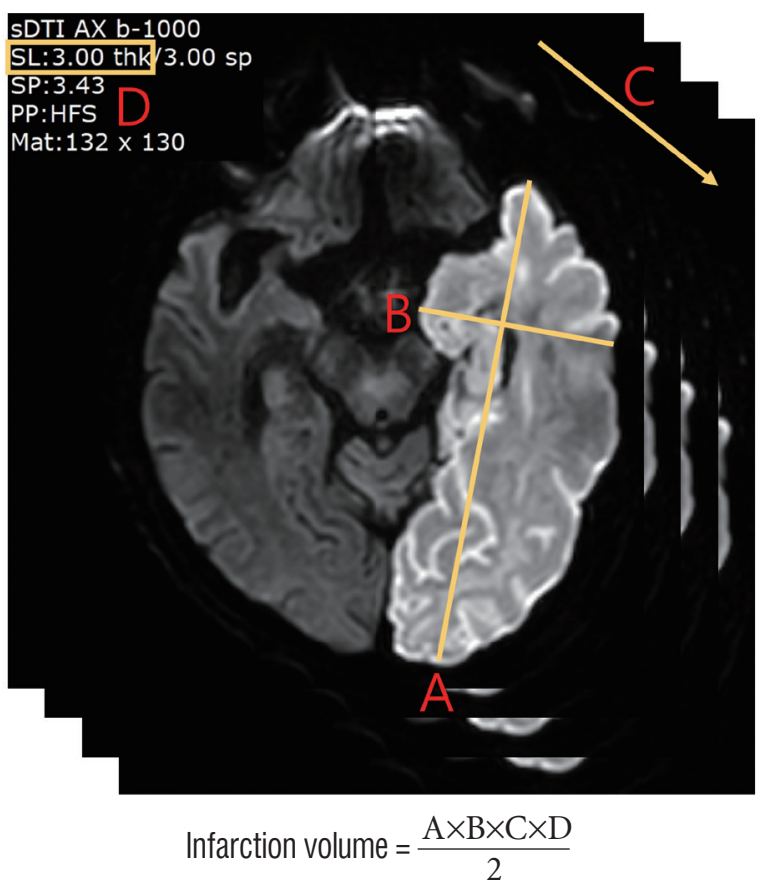

Fig. 1. Measurement of the infarction volume. $A$ : Maximum infarction diameter. B : The largest diameter perpendicular to A. C : Number of images with infarction. $D$ : Slice thickness.

\section{Clinical factors and definition of HT}

Clinical information of all patients, such as sex, age, body mass index, National Institutes of Health Stroke Scale (NIHSS) at admission, use of tissue plasminogen activator (tPA), platelet count, hypertension, diabetes, smoking, hyperlipidemia, and premedication with antithrombotics was investigated from medical records. We define HT as cases in which bleeding was observed in the acute cerebral infarction area on CT, as previously described ${ }^{9)}$.

\section{Statistical methods}

The chi-square and Student's t-tests were conducted to assess clinical differences between the two groups, divided by HT or not. The mean skull HU value was used for all analyses. The skull HU values were classified into tertile groups and analyzed. Box plots with dot plots were used to visualize the infarction volume differences between the HT groups based on sex. To determine the optimal cutoff value of infarction volume associated with HT, we performed receiver operating characteristic (ROC) curve analysis. The cumulative hazards for HT were examined using the Kaplan-Meier method classi-
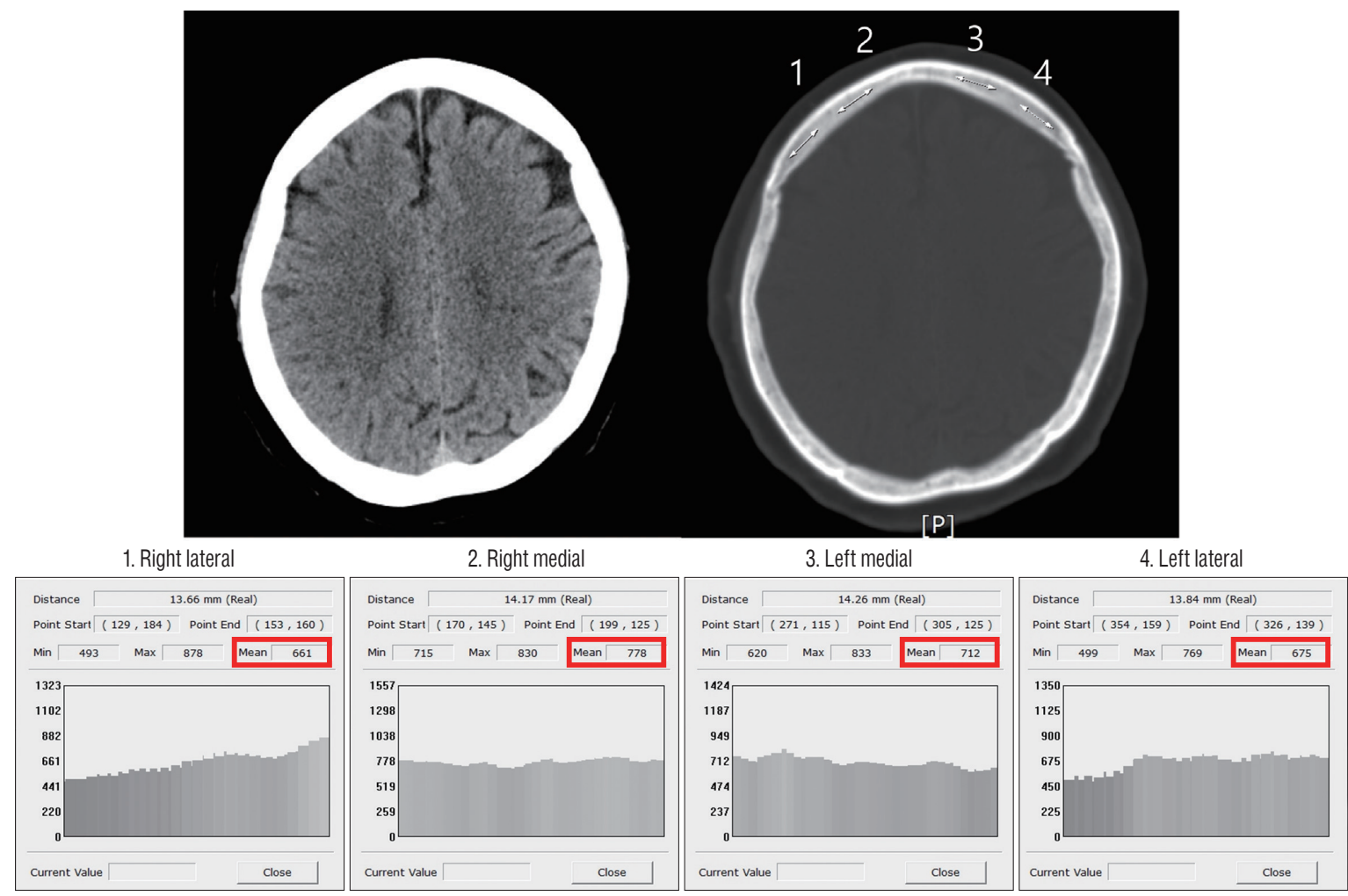

Fig. 2. Measurement of the average frontal skull Hounsfield unit (HU) value. The frontal skull HU values in the cancellous bone is measured using the "Linear histogram graph" function. 
fied by tertile groups of skull HU values. Hazard ratios with 95\% confidence intervals (CIs) were then estimated using univariate and multivariate Cox regression analyses to evaluate the independent predictive factors for HT in patients with acute cardioembolic stroke. We also performed an interaction analysis between age and skull $\mathrm{HU}$ with respect to HT.

Statistical significance was set at $p<0.05$. All statistical analyses were performed using R software, version 3.6.3 (R Foundation for Statistical Computing, Vienna, Austria) and IBM SPSS for Windows, version 24.0 (IBM Corp., Armonk, NY, USA).

\section{RESULTS}

\section{Patient characteristics}

Overall, this 11-year study enrolled 506 patients diagnosed with acute cardioembolic stroke. The HT group included 92 patients (18.2\%). The mean age of the patients was 73.6 years, and $269(53.2 \%)$ were female. There was statistical significance in NIHSS score, infarction volume, and use of tPA between the groups. Table 1 shows detailed patients information.

\section{Frontal skull HU values according to $\mathrm{HT}$ and sex}

Tables 2 and 3 show respectively the descriptive statistics of the detailed frontal skull HU values according to HT and sex.

Table 1. Clinical characteristics of patients after cerebral infarction classified according to HT in the study cohort

\begin{tabular}{|c|c|c|c|c|}
\hline Characteristic & HT (-) & HT (+) & Total & $p$-value \\
\hline Number & $414(81.8)$ & $92(18.2)$ & $506(100.0)$ & \\
\hline Sex, female & $224(54.1)$ & $45(48.9)$ & $269(53.2)$ & 0.367 \\
\hline Age (years) & $73.9 \pm 10.9$ & $72.3 \pm 10.2$ & $73.6 \pm 10.7$ & 0.193 \\
\hline BMl & $23.5 \pm 4.0$ & $23.3 \pm 3.9$ & $23.4 \pm 4.0$ & 0.640 \\
\hline NIHSS & $7.4 \pm 7.1$ & $10.9 \pm 6.4$ & $8.0 \pm 7.1$ & $<0.001$ \\
\hline Infarction volume (mL) & $36.2 \pm 78.5$ & $92.2 \pm 87.2$ & $46.4 \pm 82.9$ & $<0.001$ \\
\hline Infarction volume (mL) & $4.6(0.5-27.5)$ & $74.1(23.4-134.5)$ & $10.1(0.7-50.2)$ & $<0.001$ \\
\hline Low platelet count & $68(16.4)$ & $20(21.7)$ & $88(17.4)$ & 0.224 \\
\hline Use of tPA & $64(15.5)$ & $26(28.3)$ & $90(17.8)$ & 0.004 \\
\hline Hypertension & $250(60.4)$ & $64(69.6)$ & $314(62.1)$ & 0.101 \\
\hline Diabetes & $124(30.0)$ & $24(26.1)$ & $148(29.3)$ & 0.461 \\
\hline Current smoking & $56(13.5)$ & $17(18.5)$ & $73(14.4)$ & 0.221 \\
\hline Hyperlipidemia & $48(11.6)$ & $11(12.0)$ & $59(11.7)$ & 0.922 \\
\hline Antithrombotics & $73(17.6)$ & $11(12.0)$ & $84(16.6)$ & 0.186 \\
\hline
\end{tabular}

Values are presented as mean \pm standard deviation, median (interquartile range), or number (\%). HT : hemorrhagic transformation, BMI : body mass index, NIHSS : National Institutes of Health Stroke Scale, tPA : tissue plasminogen activator

Table 2. Descriptive statistics of frontal HU values in the study cohort classified according to HT

\begin{tabular}{lcccc}
\hline Characteristic & HT (-) & HT (+) & Total & $p$-value \\
\hline Overall mean frontal skull HU value & $673.0(507.1-872.7)$ & $636.4(475.6-825.4)$ & $670.4(503.3-864.3)$ & 0.465 \\
Overall mean frontal skull HU value & $688.8 \pm 235.8$ & $668.9 \pm 235.0$ & $685.2 \pm 235.5$ & \\
Mean HU value at each of four sites in the frontal skull & & & & \\
Right lateral & $630.5 \pm 213.6$ & $611.7 \pm 215.1$ & $627.0 \pm 213.8$ \\
Right medial & $752.4 \pm 281.2$ & $738.9 \pm 288.9$ & $750.0 \pm 282.4$ & 0.447 \\
Left medial & $744.9 \pm 281.4$ & $716.4 \pm 288.1$ & $739.7 \pm 282.5$ & 0.679 \\
Left lateral & $627.4 \pm 215.9$ & $608.7 \pm 203.9$ & $624.0 \pm 213.7$ & 0.448 \\
\hline
\end{tabular}

Values are presented as mean \pm standard deviation or median (interquartile range). HU : Hounsfield unit, HT : hemorrhagic transformation 
Hemorrhagic Transformation after Stroke I Won YD, et al.

Table 3. Descriptive statistics of frontal HU values in the study cohort classified according to sex

\begin{tabular}{|c|c|c|c|c|}
\hline Characteristic & Male & Female & Total & $p$-value \\
\hline Overall mean frontal skull HU value & $833.5(683.8-946.9)$ & $538.0(432.8-676.5)$ & $670.4(503.3-864.3)$ & $<0.001$ \\
\hline Overall mean frontal skull $\mathrm{HU}$ value & $815.6 \pm 195.0$ & $570.3 \pm 206.8$ & $685.2 \pm 235.5$ & $<0.001$ \\
\hline \multicolumn{5}{|c|}{ Mean HU value at each of four sites in the frontal skull } \\
\hline Right lateral & $715.5 \pm 192.9$ & $549.1 \pm 200.8$ & $627.0 \pm 213.8$ & $<0.001$ \\
\hline Right medial & $922.5 \pm 228.8$ & $598.0 \pm 233.7$ & $750.0 \pm 282.4$ & $<0.001$ \\
\hline Left medial & $911.4 \pm 228.4$ & $588.5 \pm 235.5$ & $739.7 \pm 282.5$ & $<0.001$ \\
\hline Left lateral & $712.9 \pm 202.1$ & $545.7 \pm 192.2$ & $624.0 \pm 213.7$ & $<0.001$ \\
\hline \multicolumn{5}{|c|}{ Classification of the skull HU based on tertile groups (HU) } \\
\hline Tertile 1 & $\leq 737.3$ & $\leq 467.5$ & $\leq 546.8$ & \\
\hline Tertile 2 & $737.3-907.3$ & $467.5-611.3$ & $546.8-815.0$ & \\
\hline Tertile 3 & $>907.3$ & $>611.3$ & $>815.0$ & \\
\hline
\end{tabular}

Values are presented as mean \pm standard deviation or median (interquartile range). $\mathrm{HU}:$ Hounsfield unit
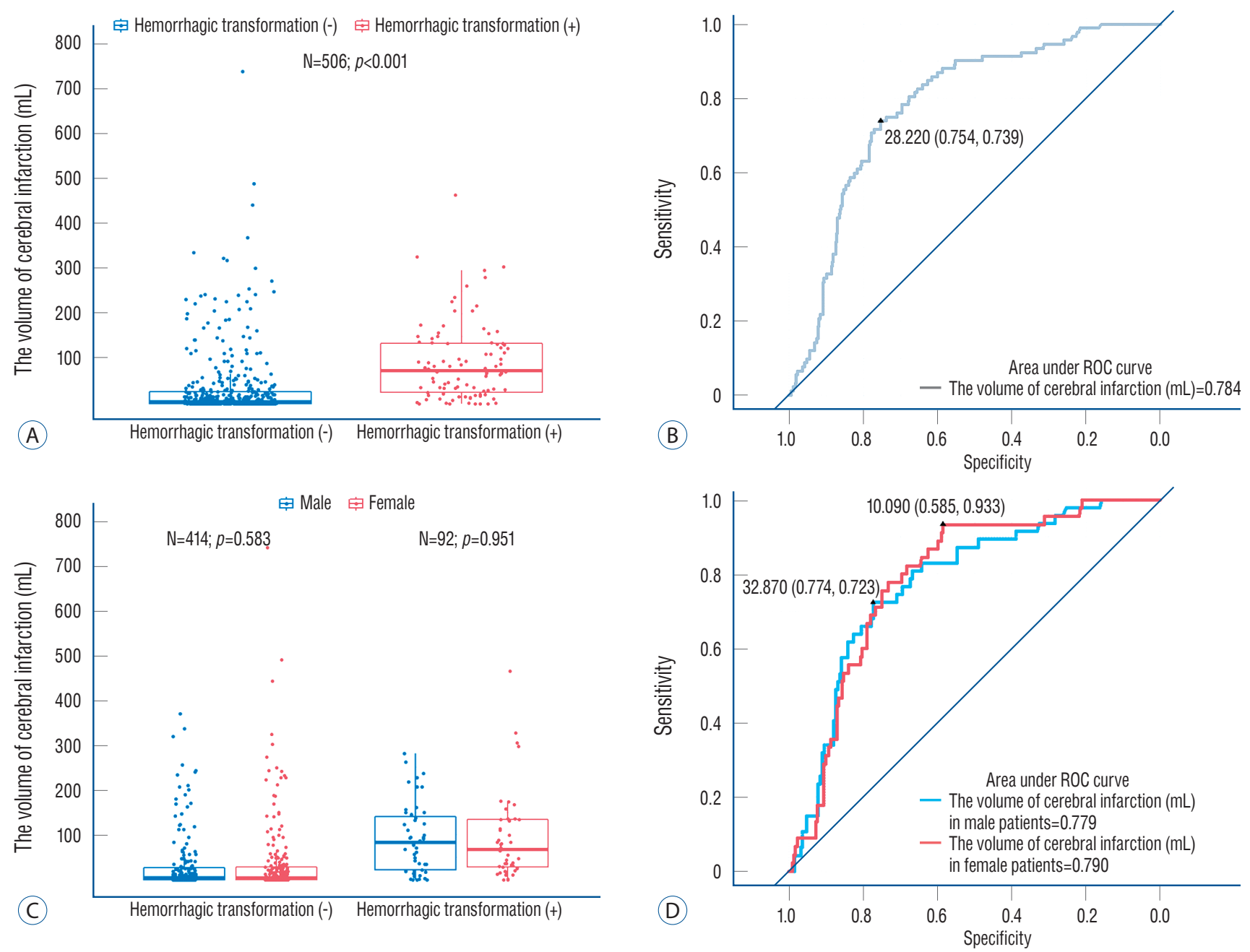

Fig. 3. Trend in infarction volumes according to hemorrhagic transformation (HT). A : Box plot with dot plots showing the trend between $\mathrm{HT}$ and infarction volumes. $\mathrm{B}$ : Receiver operating characteristic (ROC) curve for $\mathrm{HT}$ based on infarction volume. $\mathrm{C}$ : Box plot with dot plots showing the trend between $\mathrm{HT}$ and infarction volumes divided by sex. D : Receiver operating characteristic curve for HT based on infarction volume divided by sex. 
There were no significant differences in the mean frontal skull HU values according to HT (Table 2). The overall mean frontal skull HU value was 670.4; the mean frontal skull HU value was 833.5 for male and 538.0 for female patients, showing a significant difference (Table 3).

\section{Trend in infarction volume according to HT}

We observed a significant difference in the volume of cere-
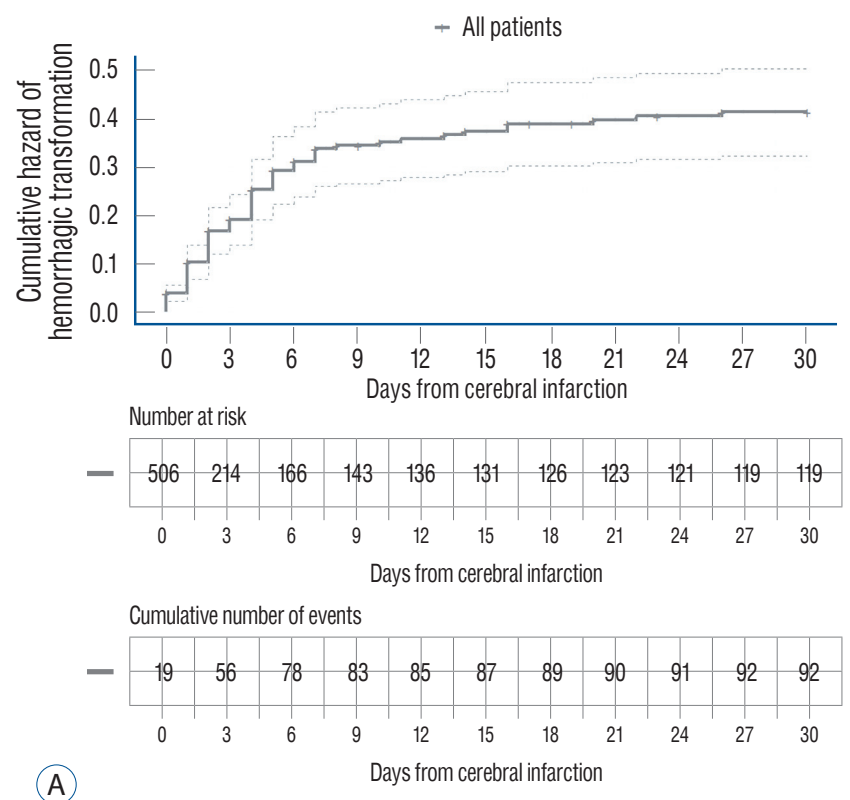

(A)

Skull HU - Tertile $1(\leq 737.25)$ - Tertile 2 (>737.25 and $\leq 907.25)$ - Tertile 3 (>907.25)
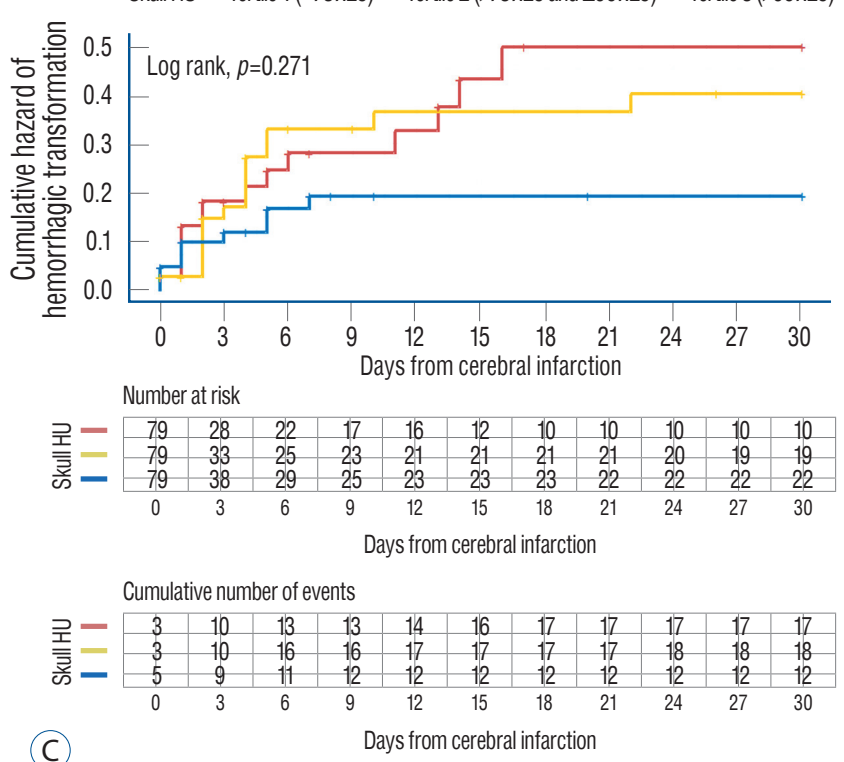

bral infarction according to HT. The HT group had a larger infarction volume than that in the none-HT group (Fig. 3A). The area under the ROC curve for the volume of cerebral infarction was 0.784 ( $p<0.001$; cutoff, $28.220 \mathrm{~mL}$ ) (Fig. 3B). There was no significant difference between the sexes in terms of the volume of cerebral infarction according to HT (Fig. 3C). The areas under the ROC curves for the volume of cerebral infarction in men and women were $0.779(p<0.001$; cutoff,
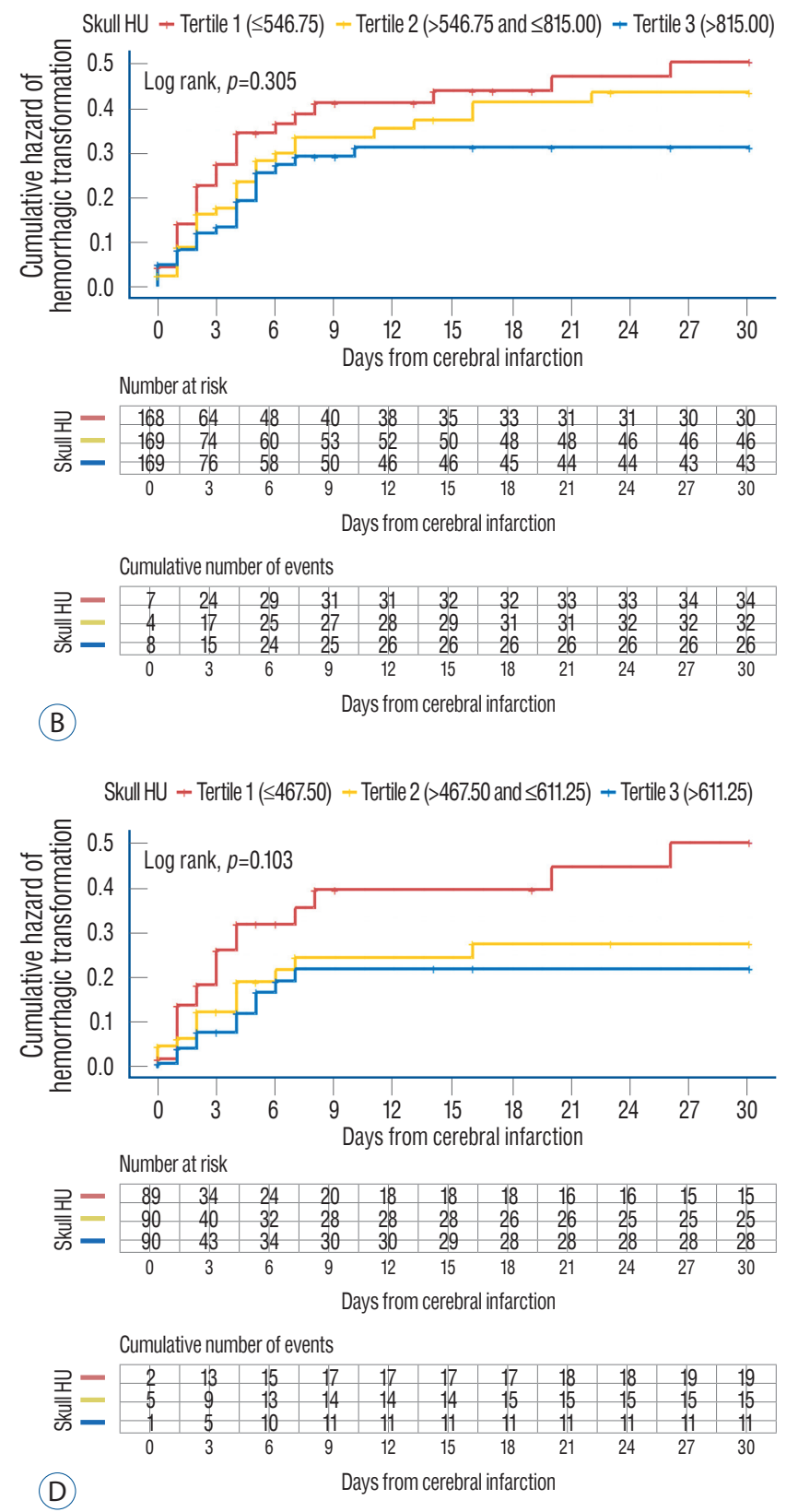

Fig. 4. Kaplan-Meier curves showing the cumulative hazards of hemorrhagic transformation (HT) after acute cardioembolic stroke. A : Cumulative hazard of HT. B : Cumulative hazards of HT according to tertile groups of frontal skull Hounsfield unit (HU) values. C : Cumulative hazards of HT according to tertile groups of frontal skull HU values in men. D : Cumulative hazards of HT according to tertile groups of frontal skull HU values in women. 
$32.870 \mathrm{~mL})$ and $0.790(p<0.001$; cutoff, $10.090 \mathrm{~mL})$, respectively (Fig. 3D).

\section{Cumulative hazard of HT after acute cardioem- bolic stroke according to skull $\mathrm{HU}$ tertile groups}

Fig. 4A showed cumulative hazard of HT after acute cardioembolic stroke. Although not statistically significant, the first tertile of skull $\mathrm{HU}$ values showed greater HT within 1 month

Table 4. Univariate and multivariate Cox regression analyses of hemorrhagic transformation after acute cerebral infarction based on predictive factors

\begin{tabular}{|c|c|c|c|c|}
\hline \multirow{2}{*}{ Variable } & \multicolumn{2}{|c|}{ Univariate } & \multicolumn{2}{|c|}{ Multivariate } \\
\hline & $\mathrm{HR}(95 \% \mathrm{Cl})$ & $p$-value & HR $(95 \% \mathrm{Cl})$ & $p$-value \\
\hline \multicolumn{5}{|l|}{ Sex } \\
\hline Male & Reference & & Reference & \\
\hline Female & $0.82(0.54-1.23)$ & 0.328 & $0.76(0.46-1.26)$ & 0.282 \\
\hline Age, per 1-year increase & $0.99(0.97-1.01)$ & 0.195 & $0.97(0.95-1.00)$ & 0.020 \\
\hline BMI, per 1-unit increase & $0.98(0.93-1.04)$ & 0.548 & $0.96(0.91-1.01)$ & 0.126 \\
\hline NIHSS at admission, per 1-unit increase & $1.05(1.02-1.07)$ & $<0.001$ & $1.04(1.01-1.07)$ & 0.024 \\
\hline Infarction volume, per $1 \mathrm{~mL}$ increment & $1.01(1.00-1.01)$ & $<0.001$ & $1.00(1.00-1.01)$ & $<0.001$ \\
\hline \multicolumn{5}{|l|}{ Use of tPA } \\
\hline No & Reference & & Reference & \\
\hline Yes & $1.55(0.99-2.45)$ & 0.058 & $1.15(0.71-1.85)$ & 0.569 \\
\hline \multicolumn{5}{|l|}{ Platelet count } \\
\hline Normal or high & Reference & & Reference & \\
\hline Low (<150000 per microliter) & $1.53(0.93-2.51)$ & 0.094 & $1.32(0.79-2.20)$ & 0.296 \\
\hline \multicolumn{5}{|l|}{ Tertile groups of mean frontal skull HU } \\
\hline Tertile $1(\leq 546.8)$ & $1.48(0.89-2.46)$ & 0.136 & $2.12(1.13-3.98)$ & 0.020 \\
\hline Tertile 2 (>546.8 and $\leq 815.0$ ) & $1.20(0.71-2.01)$ & 0.496 & $1.65(0.94-2.91)$ & 0.080 \\
\hline Tertile $3(>815.0)$ & Reference & & Reference & \\
\hline \multicolumn{5}{|l|}{ Hypertension } \\
\hline No & Reference & & Reference & \\
\hline Yes & $1.33(0.86-2.08)$ & 0.205 & $1.66(1.03-2.69)$ & 0.038 \\
\hline \multicolumn{5}{|l|}{ Diabetes } \\
\hline No & Reference & & Reference & \\
\hline Yes & $0.82(0.51-1.30)$ & 0.392 & $0.88(0.54-1.43)$ & 0.607 \\
\hline \multicolumn{5}{|l|}{ Current smoking } \\
\hline No & Reference & & Reference & \\
\hline Yes & $1.39(0.82-2.36)$ & 0.217 & $1.13(0.61-2.09)$ & 0.695 \\
\hline \multicolumn{5}{|l|}{ Hyperlipidemia } \\
\hline No & Reference & & Reference & \\
\hline Yes & $0.98(0.52-1.84)$ & 0.945 & $0.92(0.48-1.76)$ & 0.806 \\
\hline \multicolumn{5}{|l|}{ Antithrombotics } \\
\hline No & Reference & & Reference & \\
\hline Yes & $0.63(0.33-1.18)$ & 0.147 & $0.66(0.35-1.27)$ & 0.210 \\
\hline
\end{tabular}

HR : hazard ratio, Cl : confidence interval, BMI : body mass index, NIHSS : National Institutes of Health Stroke Scale, tPA: tissue plasminogen activator, HU : Hounsfield unit 
Table 5. Univariate and multivariate Cox regression analyses of hemorrhagic transformation after acute cerebral infarction based on predictive factors in the male group

\begin{tabular}{|c|c|c|c|c|}
\hline \multirow{2}{*}{ Variable } & \multicolumn{2}{|c|}{ Univariate } & \multicolumn{2}{|c|}{ Multivariate } \\
\hline & HR $(95 \% \mathrm{Cl})$ & $p$-value & $\mathrm{HR}(95 \% \mathrm{Cl})$ & $p$-value \\
\hline Age, per 1-year increase & $0.98(0.96-1.01)$ & 0.188 & $0.97(0.94-1.01)$ & 0.126 \\
\hline BMI, per 1-unit increase & $1.02(0.95-1.10)$ & 0.581 & $0.98(0.91-1.07)$ & 0.669 \\
\hline NIHSS at admission, per 1-unit increase & $1.04(1.00-1.07)$ & 0.031 & $1.04(0.99-1.08)$ & 0.137 \\
\hline Infarction volume, per $1 \mathrm{~mL}$ increment & $1.01(1.00-1.01)$ & $<0.001$ & $1.00(1.00-1.01)$ & 0.036 \\
\hline \multicolumn{5}{|l|}{ Use of tPA } \\
\hline No & Reference & & Reference & \\
\hline Yes & $1.68(0.91-3.11)$ & 0.098 & $1.17(0.59-2.32)$ & 0.653 \\
\hline \multicolumn{5}{|l|}{ Platelet count } \\
\hline Normal or high & Reference & & Reference & \\
\hline Low (<150000 per microliter) & $1.29(0.67-2.49)$ & 0.445 & $1.52(0.76-3.06)$ & 0.238 \\
\hline \multicolumn{5}{|l|}{ Tertile groups of mean frontal skull HU } \\
\hline Tertile $1(\leq 737.3)$ & $1.77(0.84-3.71)$ & 0.132 & $1.87(0.87-4.02)$ & 0.111 \\
\hline Tertile 2 (>737.3 and $\leq 907.3$ ) & $1.58(0.76-3.29)$ & 0.218 & $1.70(0.79-3.66)$ & 0.173 \\
\hline Tertile 3 (>907.3) & Reference & & Reference & \\
\hline \multicolumn{5}{|l|}{ Hypertension } \\
\hline No & Reference & & Reference & \\
\hline Yes & $1.43(0.78-2.61)$ & 0.249 & $1.80(0.92-3.53)$ & 0.087 \\
\hline \multicolumn{5}{|l|}{ Diabetes } \\
\hline No & Reference & & Reference & \\
\hline Yes & $0.88(0.45-1.74)$ & 0.718 & $0.96(0.48-1.95)$ & 0.916 \\
\hline \multicolumn{5}{|l|}{ Current smoking } \\
\hline No & Reference & & Reference & \\
\hline Yes & $1.47(0.81-2.67)$ & 0.205 & $1.36(0.68-2.71)$ & 0.382 \\
\hline \multicolumn{5}{|l|}{ Hyperlipidemia } \\
\hline No & Reference & & Reference & \\
\hline Yes & $0.95(0.40-2.25)$ & 0.913 & $1.01(0.40-2.59)$ & 0.977 \\
\hline \multicolumn{5}{|l|}{ Antithrombotics } \\
\hline No & Reference & & Reference & \\
\hline Yes & $0.71(0.30-1.67)$ & 0.434 & $0.73(0.29-1.80)$ & 0.488 \\
\hline
\end{tabular}

HR : hazard ratio, Cl : confidence interval, BMI : body mass index, NIHSS : National Institutes of Health Stroke Scale, tPA: tissue plasminogen activator, HU : Hounsfield unit

after acute cerebral infarction compared to the second and third tertiles (Fig. 4B). Even when sepaearwly analyzed for males and females, the first tertile of skull HU value tended to have a higher risk of HT than the second and third teitiles but had no statistical significe (Fig. 4C and D).

\section{Independent predictive factors for HT in acute cardioembolic stroke}

Multivariate Cox regression analysis showed that skull HU within the first tertile was an independent predictor of $\mathrm{HT}$ after acute cardioembolic stroke compared to those in the highest tertile group (hazard ratio, 2.12; 95\% CI, 1.13-3.98; $p=0.020$ ) (Table 4). The other independent predictors of HT were young- 
Hemorrhagic Transformation after Stroke I Won YD, et al.

Table 6. Univariate and multivariate Cox regression analyses of hemorrhagic transformation after acute cerebral infarction based on predictive factors in the female group

\begin{tabular}{|c|c|c|c|c|}
\hline \multirow{2}{*}{ Variable } & \multicolumn{2}{|c|}{ Univariate } & \multicolumn{2}{|c|}{ Multivariate } \\
\hline & $\mathrm{HR}(95 \% \mathrm{CI})$ & $p$-value & $\mathrm{HR}(95 \% \mathrm{Cl})$ & $p$-value \\
\hline Age, per 1-year increase & $1.00(0.97-1.03)$ & 0.960 & $0.97(0.93-1.01)$ & 0.093 \\
\hline BMI, per 1-unit increase & $0.95(0.89-1.02)$ & 0.187 & $0.96(0.88-1.03)$ & 0.255 \\
\hline NIHSS at admission, per 1-unit increase & $1.06(1.02-1.10)$ & 0.002 & $1.04(0.99-1.08)$ & 0.122 \\
\hline Infarction volume, per $1 \mathrm{~mL}$ increment & $1.01(1.00-1.01)$ & $<0.001$ & $1.01(1.00-1.01)$ & 0.001 \\
\hline \multicolumn{5}{|l|}{ Use of tPA } \\
\hline No & Reference & & Reference & \\
\hline Yes & $1.38(0.70-2.73)$ & 0.353 & $1.32(0.64-2.73)$ & 0.458 \\
\hline \multicolumn{5}{|l|}{ Platelet count } \\
\hline Normal or high & Reference & & Reference & \\
\hline Low (<150000 per microliter) & $1.84(0.85-3.95)$ & 0.121 & $1.24(0.52-2.92)$ & 0.628 \\
\hline \multicolumn{5}{|l|}{ Tertile groups of mean frontal skull HU } \\
\hline Tertile $1(\leq 467.5)$ & $2.15(1.02-4.52)$ & 0.044 & $2.55(1.07-6.09)$ & 0.035 \\
\hline Tertile 2 (>467.5 and $\leq 611.3$ ) & $1.42(0.65-3.10)$ & 0.375 & $1.29(0.54-3.10)$ & 0.569 \\
\hline Tertile 3 (>611.3) & Reference & & Reference & \\
\hline \multicolumn{5}{|l|}{ Hypertension } \\
\hline No & Reference & & Reference & \\
\hline Yes & $1.29(0.67-2.50)$ & 0.452 & $1.51(0.75-3.04)$ & 0.246 \\
\hline \multicolumn{5}{|l|}{ Diabetes } \\
\hline No & Reference & & Reference & \\
\hline Yes & $0.79(0.42-1.52)$ & 0.485 & $0.90(0.45-1.79)$ & 0.769 \\
\hline \multicolumn{5}{|l|}{ Current smoking } \\
\hline No & Reference & & Reference & \\
\hline Yes & $0.05(0.00-346.59)$ & 0.503 & $0.00(0.00-0.00)$ & 0.971 \\
\hline \multicolumn{5}{|l|}{ Hyperlipidemia } \\
\hline No & Reference & & Reference & \\
\hline Yes & $0.99(0.39-2.52)$ & 0.989 & $0.93(0.35-2.47)$ & 0.878 \\
\hline \multicolumn{5}{|l|}{ Antithrombotics } \\
\hline No & Reference & & Reference & \\
\hline Yes & $0.55(0.22-1.40)$ & 0.210 & $0.72(0.27-1.90)$ & 0.500 \\
\hline
\end{tabular}

HR : hazard ratio, Cl : confidence interval, BMI : body mass index, NIHSS : National Institutes of Health Stroke Scale, tPA: tissue plasminogen activator, HU : Hounsfield unit

er age, higher NIHSS score, larger infarction volume, and hypertension. In men, a larger infarction volume was the only independent predictor of HT (Table 5). However, among women, the first tertile of skull HU (hazard ratio, 2.55; 95\% CI, 1.07$6.09 ; p=0.035)$ and larger infarction volume were independent predictors of HT (Table 6).

\section{Interaction analysis between age and skull HU values with respect to $\mathrm{HT}$}

Because age is correlated with BMD, we further performed an interaction analysis between age and skull HU with respect to HT in patients with after acute cardioembolic stroke. However, we observed no significant interactions between age and skull HU values. Similarly, the analysis classified by sex also 

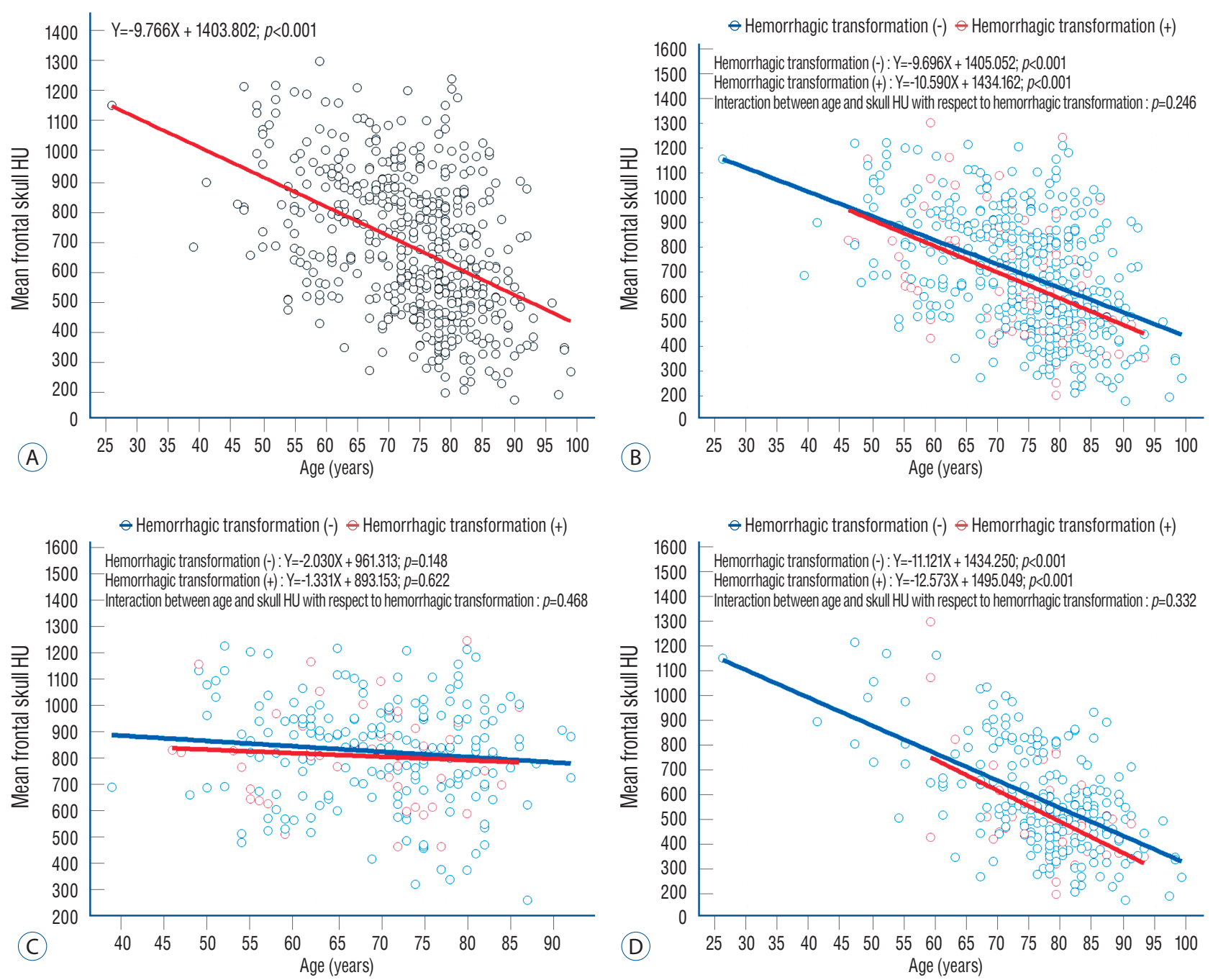

Fig. 5. Interaction plots between age and frontal skull Hounsfield units (HUs) with respect to hemorrhagic transformation (HT) after acute cardioembolic stroke. A : Scatter plots with linear regression lines depicting the associations between age and mean frontal skull HU values. B : Interaction between age and frontal skull HU with respect to HT. C : Interaction between age and frontal skull HU with respect to HT in men. D: Interaction between age and frontal skull HU with respect to $\mathrm{HT}$ in women.

showed no significant interactions between age and skull HU values (Fig. 5).

\section{DISCUSSION}

The results of our study show that a low frontal skull HU is associated with a higher risk of HT in patients with acute cardioembolic stroke compared to a higher frontal skull HU. Therefore, a low BMD state or osteoporotic condition may be independently associated with HT in the clinical course of acute cardioembolic stroke. In addition, when we classified patients by sex, we observed that only women showed a sig- nificant association between low skull HU and HT development in the multivariate analysis.

Osteoporosis is a systemic disease that is strongly associated with the genetic components of type 1 collagen, such as COL1A1 and COL1A2. Cancellous bone structures in the skull may also be affected by osteoporotic conditions. Previous studies have shown that the HU value of the cancellous bone can be useful in predicting the osteoporotic condition because this value in a specific area on CT scans is strongly related to the $\mathrm{T}$ score ${ }^{6,26)}$. We also reported a strong association between skull HU values and T-scores and that the HU values of the frontal skull cancellous bone predicted osteoporotic status ${ }^{13,31)}$. Type 1 collagen is a major component of bone. Genetic muta- 
tions in type 1 collagen can lead to osteoporosis or low BMD. Among the many components that make up blood vessels, smooth muscle is also composed of type 1 collagen $^{24)}$. Smooth muscles control artery contraction and relaxation; thus, lack or degeneration of smooth muscles can lead to secondary vascular changes, including fibrinoid necrosis and aneurysm-like vasodilatation that can cause vessel fragility and rupture ${ }^{10,29)}$. Osteogenesis imperfecta $(\mathrm{OI})$ caused by mutations in type 1 collagen genes is also associated with hemorrhagic diathesis, and is also hypothesized to be associated with vascular fragility ${ }^{12,21)}$. In addition, abnormalities in the cerebral arterial system have been reported in $\mathrm{OI}^{2)}$. Some patients with $\mathrm{OI}$ show distal internal cerebral artery stenosis, middle cerebral artery occlusion, and moyamoya-like collateral arising from the lenticulostriate arteries. These findings were interpreted as vasculopathic changes secondary to vascular fragility caused by collagen abnormalities in OI. Therefore, our results can be explained by the following hypothetical mechanism. Reperfusion occurs after acute cerebral infarction; moreover, the higher the fragility of blood vessels due to the weakened integrity of smooth muscle, the lower the ability to withstand reperfusion, thus increasing the possibility of HT. Among the women in this study, those with a low frontal skull HU value showed an approximately 2.6-fold higher risk of HT after cardiac embolism infarction compared to those with a higher frontal skull HU value after adjusting for other predictive factors, including age. In men, however, the difference between the frontal skull HU values and HT was not significant. Women aged 50 and over are reported to have four times higher rates of osteoporosis than men ${ }^{3)}$. We also found that women had a higher rate of possible osteoporosis than men. The mean frontal skull HU value of men was significantly higher than that of women (833.5 vs. 538.0), and this result is likely not to have been analyzed as a risk factor for HT because men are more likely to have less osteoporosis than women.

However, in a recent study with the parenchymal type HT as the only dependent variable, contrary to our findings, only men showed a significant association between hypothetical osteoporosis and parenchymal type HT development in the multivariate Cox analysis ${ }^{30)}$. Although, more research is needed in the future, we believe that men with osteoporosis may be more vulnerable to severe HT development after cardioembolic stroke than women. Males with ICH was more associated with risk of hematoma expansion and mortality rate compared to females $^{20)}$. In addition, males showed more osteoporosis-related complications and a higher mortality rate than females in osteoporotic fractures ${ }^{3,5)}$. Furthermore, estrogen is known to have an important role in maintaining adequate cerebral perfusion and this may cause sex differences in hemorrhage expansion and mortality ${ }^{25)}$.

Our findings suggest that, additional possible predictive factors for HT after acute cerebral infarction include younger age, higher NIHSS score at admission, hypertension and larger infarction volume. These variables are predictive factors of HT after acute cerebral infarction, as reported previous$\mathrm{ly}^{7,8,14,15,17,18,23,27)}$.

Our study has some limitations. First, the time between subsequent CT images was irregular due to the retrospective nature of the study. In particular, in the case of asymptomatic $\mathrm{HT}$, there may be an error in time between the occurrence of HT and the CT scan. Second, true T scores were not available in patients with acute cerebral infarction because the assessment of BMD status is generally not required. Moreover, while the cancellous bone HU values of the frontal skull are strongly correlated with the actual BMD, they may not directly reflect the exact $\mathrm{T}$ score. Third, measurement errors may have occurred. However, we enlarged all brain CT images for HU measurement to exclude cortical bones; moreover, patients without measurable frontal bone cancellous bones were excluded from the study. Fourth, it is well known that BBB disruption is crucial for HT development ${ }^{4}$. Therefore, our findings, which show the association between weakened vascular smooth muscle integrity and HT, should be validated by future study. Finally, we analyzed only Korean patients, which may limit the generalizability of the results owing to genetic differences between regions and races that affect BMD. Therefore, further research is required to verify these results.

\section{CONCLUSION}

In conclusion, the results of our study suggest an association between osteoporotic conditions and HT development after acute cerebral infarction with cardiac embolism. This association was more prominent in women than in men. Our findings may be useful for predicting HT in patients with acute cerebral infarction using a convenient method to measure the $\mathrm{HU}$ value of the cancellous bone of the frontal skull using brain CT imaging. However, confirming these initial results 
require further studies, including different types of acute cerebral infarction. Our findings may help improve our understanding of the underlying mechanisms of the relationship between HT and BMD in acute cerebral infarction.

\section{CONFLICTS OF INTEREST}

No potential conflict of interest relevant to this article was reported.

\section{INFORMED CONSENT}

This type of study does not require informed consent.

\section{AUTHOR CONTRIBUTIONS}

\author{
Conceptualization : MHH, YDW \\ Data curation : YDW \\ Formal analysis : $\mathrm{MHH}$ \\ Funding acquisition : $\mathrm{MHH}$ \\ Methodology : MHH, YDW \\ Project administration: JHC \\ Visualization: $\mathrm{MHH}$ \\ Writing - original draft : YDW, $\mathrm{MHH}$ \\ Writing - review \& editing: JMK, JIR, SHK, JHC
}

\section{ORCID}

Yu Deok Won https://orcid.org/0000-0002-8822-6631

Jae-Min Kim https://orcid.org/0000-0003-4880-4553 Je-Il Ryu https://orcid.org/0000-0002-4785-9475 Seong-Ho Koh https://orcid.org/0000-0001-5419-5761 Myung-Hoon Han https://orcid.org/0000-0003-1728-5017 Jin-Hwan Cheong https://orcid.org/0000-0002-0022-312X

\section{References}

1. Adams HP Jr, Bendixen BH, Kappelle LJ, Biller J, Love BB, Gordon DL, et al. : Classification of subtype of acute ischemic stroke. Definitions for use in a multicenter clinical trial. TOAST. Trial of Org 10172 in acute stroke treatment. Stroke 24 : 35-41, 1993

2. Albayram S, Kizilkilic O, Yilmaz H, Tuysuz B, Kocer N, Islak C : Abnormalities in the cerebral arterial system in osteogenesis imperfecta. AJNR Am J Neuroradiol 24 : 748-750, 2003

3. Alswat KA : Gender disparities in osteoporosis. J Clin Med Res 9 : 382-387, 2017

4. Arba F, Rinaldi C, Caimano D, Vit F, Busto G, Fainardi E : Blood-brain barrier disruption and hemorrhagic transformation in acute ischemic stroke: systematic review and meta-analysis. Front Neurol 11 : 594613, 2021

5. Center JR, Nguyen TV, Schneider D, Sambrook PN, Eisman JA : Mortality after all major types of osteoporotic fracture in men and women: an observational study. Lancet 353 : 878-882, 1999

6. Choi MK, Kim SM, Lim JK : Diagnostic efficacy of Hounsfield units in spine $\mathrm{CT}$ for the assessment of real bone mineral density of degenerative spine: correlation study between T-scores determined by DEXA scan and Hounsfield units from CT. Acta Neurochir (Wien) 158 : 14211427,2016

7. Cucchiara B, Kasner SE, Tanne D, Levine SR, Demchuk A, Messe SR, et al. : Factors associated with intracerebral hemorrhage after thrombolytic therapy for ischemic stroke: pooled analysis of placebo data from the Stroke-Acute Ischemic NXY Treatment (SAINT) I and SAINT II trials. Stroke 40 : 3067-3072, 2009

8. Cucchiara B, Tanne D, Levine SR, Demchuk AM, Kasner S : A risk score to predict intracranial hemorrhage after recombinant tissue plasminogen activator for acute ischemic stroke. J Stroke Cerebrovasc Dis 17 : 331-333, 2008

9. Fiorelli M, Bastianello S, von Kummer R, del Zoppo GJ, Larrue V, Lesaf-

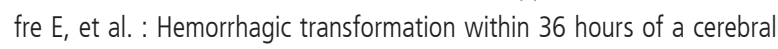
infarct: relationships with early clinical deterioration and 3-month outcome in the European Cooperative Acute Stroke Study I (ECASS I) cohort. Stroke 30 : 2280-2284, 1999

10. Frösen J, Joutel $A$ : Smooth muscle cells of intracranial vessels: from development to disease. Cardiovasc Res 114 : 501-512, 2018

11. Gajko-Galicka A : Mutations in type I collagen genes resulting in osteogenesis imperfecta in humans. Acta Biochim Pol 49 : 433-441, 2002

12. Gooijer K, Rondeel JMM, van Dijk FS, Harsevoort AGJ, Janus GJM, Franken AAM : Bleeding and bruising in osteogenesis imperfecta: International Society on Thrombosis and Haemostasis bleeding assessment tool and haemostasis laboratory assessment in 22 individuals. Br J Haematol $187: 509-517,2019$

13. Han MH, Won YD, Na MK, Kim CH, Kim JM, Ryu Jl, et al. : Association between possible osteoporosis and shunt-dependent hydrocephalus after subarachnoid hemorrhage. Stroke 49 : 1850-1858, 2018

14. Kerenyi L, Kardos L, Szász J, Szatmári S, Bereczki D, Hegedüs K, et al. : Factors influencing hemorrhagic transformation in ischemic stroke: a clinicopathological comparison. Eur J Neurol 13 : 1251-1255, 2006

15. Kidwell CS, Saver JL, Carneado J, Sayre J, Starkman S, Duckwiler G, et al. : Predictors of hemorrhagic transformation in patients receiving intraarterial thrombolysis. Stroke 33 : 717-724, 2002 
16. Kothari RU, Brott T, Broderick JP, Barsan WG, Sauerbeck LR, Zuccarello $M$, et al. : The $A B C s$ of measuring intracerebral hemorrhage volumes. Stroke 27 : 1304-1305, 1996

17. Lansberg MG, Albers GW, Wijman CA : Symptomatic intracerebral hemorrhage following thrombolytic therapy for acute ischemic stroke: a review of the risk factors. Cerebrovasc Dis 24 : 1-10, 2007

18. Larrue V, von Kummer RR, Müller A, Bluhmki E : Risk factors for severe hemorrhagic transformation in ischemic stroke patients treated with recombinant tissue plasminogen activator: a secondary analysis of the European-Australasian Acute Stroke Study (ECASS II). Stroke 32 : 438441, 2001

19. Lodder J, Krijne-Kubat B, Broekman J : Cerebral hemorrhagic infarction at autopsy: cardiac embolic cause and the relationship to the cause of death. Stroke 17 : 626-629, 1986

20. Marini S, Morotti A, Ayres AM, Crawford K, Kourkoulis CE, Lena UK, et al. : Sex differences in intracerebral hemorrhage expansion and mortality. J Neurol Sci 379 : 112-116, 2017

21. Mondal RK, Mann U, Sharma M : Osteogenesis imperfecta with bleeding diathesis. Indian J Pediatr 70 : 95-96, 2003

22. Moulin T, Crépin-Leblond T, Chopard JL, Bogousslavsky J : Hemorrhagic infarcts. Eur Neurol 34 : 64-77, 1994

23. Paciaroni M, Agnelli G, Corea F, Ageno W, Alberti A, Lanari A, et al. : Early hemorrhagic transformation of brain infarction: rate, predictive factors, and influence on clinical outcome: results of a prospective multicenter study. Stroke 39 : 2249-2256, 2008

24. Ponticos M, Partridge T, Black CM, Abraham DJ, Bou-Gharios G : Regu- lation of collagen type I in vascular smooth muscle cells by competition between Nkx2.5 and SEF1/ZEB1. Mol Cell Biol 24 : 6151-6161, 2004

25. Roof RL, Hall ED : Estrogen-related gender difference in survival rate and cortical blood flow after impact-acceleration head injury in rats. J Neurotrauma 17 : 1155-1169, 2000

26. Schreiber JJ, Anderson PA, Rosas HG, Buchholz AL, Au AG : Hounsfield units for assessing bone mineral density and strength: a tool for osteoporosis management. J Bone Joint Surg Am 93 : 1057-1063, 2011

27. Selim M, Fink JN, Kumar S, Caplan LR, Horkan C, Chen Y, et al. : Predictors of hemorrhagic transformation after intravenous recombinant tissue plasminogen activator: prognostic value of the initial apparent diffusion coefficient and diffusion-weighted lesion volume. Stroke 33 : 20472052, 2002

28. Sims $J R$, Gharai $L R$, Schaefer $P W$, Vangel $M$, Rosenthal ES, Lev $M H$, et al. : $A B C / 2$ for rapid clinical estimate of infarct, perfusion, and mismatch volumes. Neurology 72 : 2104-2110, 2009

29. Winkler DT, Bondolfi L, Herzig MC, Jann L, Calhoun ME, Wiederhold KH, et al. : Spontaneous hemorrhagic stroke in a mouse model of cerebral amyloid angiopathy. J Neurosci 21 : 1619-1627, 2001

30. Won YD, Kim JM, Cheong JH, Ryu Jl, Koh SH, Han MH : Effect of possible osteoporosis on parenchymal-type hemorrhagic transformation in patients with cardioembolic stroke. J Clin Med 10 : 2526, 2021

31. Won YD, Na MK, Kim CH, Kim JM, Cheong JH, Ryu JI, et al. : The frontal skull Hounsfield unit value can predict ventricular enlargement in patients with subarachnoid haemorrhage. Sci Rep 8 : 10178, 2018 